\title{
Twin Chaos Simultaneous Asynchronous Oscillations of Chaos
}

\author{
Katsunori Suzuki†, Yoshifumi Nishioł and Shinsaku Mori† \\ † Dept. of Electrical Engineering, Keio University \\ 3-14-1 Hiyoshi, Kouhoku, Yokohama, 223 Japan \\ Tel: +81-45-563-1141 Email: suzuki@mori.elec.keio.ac.jp \\ $\ddagger$ Dept. of Electrical and Electronic Engineering, University of Tokushima \\ 2-1 Minami-Josanjima, Tokushima, 770 Japan \\ Email: nishio@ee.tokushima-u.ac.jp
}

\begin{abstract}
In this study, simultaneous asynchronous oscillations of chaos observed in a simple autonomous circuit are investigated by both of circuit experiments and computer calculations. This circuit consists of two nonlinear subcircuits having different oscillation frequencies coupled by one linear negative resistor. In the case that oscillation frequencies of two subcircuits are quite different, both subcircuits exhibit asynchronous chaos simultaneously. Namely, the oscillation is almost divided into two different kinds of chaos, one includes many lower frequency components and the other one includes many higher frequency components. We call this interesting phenomenon as twin chaos. Moreover, as the different between oscillation frequencies of two subcircuits decreases, two difference kinds of chaos interfere mutually. Finally, twin chaos disappears and chaos which is the same as the case of one subcircuit appears due to mutual leading-in.
\end{abstract}

\section{IN'TRODUCTION}

There have been many investigations of simultaneous asynchronous oscillators with many degrees of freedom since the subject had been introduced by van der Pol who considered a lumped oscillator with two degrees of freedom [1]-[5]. Schaffner has considered simultaneous asynchronous multifrequencies oscillator with fifthpower negative nonlinear $v-i$ characteristics [3]. He showed that nonresonant two oscillations are possible in such a system but are not self-starting. Disman and Edison have investigated that self-biasing effects in class-C oscillator can permit self-starting nonresonant two oscillations [4]. Saito et al have considered simultaneous asynchronous oscillations in negative conductance oscillator with nonlinear resonators [5].

On the other hand, in the study of autonomous chaotic circuits, three dimensional systems have reasonably well investigated [6]-[9]. Inaba et al have investigated an autonomous chaotic circuit constructed from three memory elements, one linear negative resistor and one diode [7], [8]. In the case that we regard the diode as an ideal limiter [9], the switching operation of the diode changes the oscillation frequency in the circuit considerably and this effect causes a stretching and folding mechanism. We have explained the physical mechanism of the generation of chaos by changing oscillation frequency. Recently, four or higher dimensional chaotic systems have begun to be investigated (see Refs.[10], [11] and references therein). Although a kind of simultaneous asynchronous oscillator is a good example of higherdimensional systems, studies on chaotic phenomena observed in such systems have not been reported as far as we know.

In this study, simultaneous asynchronous oscillations of chaos observed in a simple autonomous circuit based on one proposed by Inaba et al [7] are investigated. 'l'he circuit model consists of two subcircuits construcled from three memory elements and one nonlinear resistor and only one negative conductance. By idealizing the diode [9], the circuit dynamics are simplified. In our system, two kinds of chaos can be observed. One chaos includes many higher frequency components and the other one includes many lower frequency components. In the case that our circuit exhibits asynchronous chaos simultaneously, we obtain the spectral characteristics having two crests on lower frequency band and higher frequency band. We confirm the existence of simultaneous two chaotic oscillations in this spectrum. We call this phenomenon as twin chaos. Further, we carry out circuit experiments in the case that the difference of oscillation frequencies in both of subcircuits is small. Although the effect of mutual leading-in occurs in the system, we can also observe almost twin chaos. We believe that chaos generated in our systen is almost divided into two kinds of chaos on lower frequency band and higher frequency 
band and this result indicates some effective suggestions to make clear the chaotic phenomena in higher dimensional systems coupled same chaotic circuits. 'The circuit, has the broad spectrum and the spectrum is controlled simply and arbitrarily by only varying values of memory elements. Therefore, we expect that it is possible that this system applicates a noise generator.

\section{CIRCUIT MODEL}

Circuit model is shown in Fig. 1. 'This twin chaos generator is based on the circuit proposed by Inaba ef al [7] and consists of two subcircuits coupled in series and one negative conductance. 'The subcircuit consists of three memory elements and one diode.

$\Lambda$ first, the $i-v$ characteristics of the diode is approx imated by following 2-segment piecewise-linear function.

$$
v_{d\left(i_{d}\right)}=\frac{r_{d}}{2}\left(i_{d}+\frac{E}{r_{d}}-\left|i_{d}-\frac{E}{r_{d}}\right|\right)
$$

All diodes included in our circuit are described by liq. (1) because of same threshold voltage $E$. Using the following variables and paramelers,

$$
\begin{gathered}
t=\sqrt{L_{11} C_{1}} \tau, \quad " .=\frac{d}{d \tau}, \quad \alpha=g \sqrt{\frac{L_{11}}{C_{1}}}, \\
\beta_{1}=\frac{L_{11}}{L_{12}}, \quad \beta_{2}=\frac{L_{11}}{L_{21}}, \quad \beta_{3}=\frac{L_{11}}{L_{22}}, \\
\gamma=\frac{C_{1}}{C_{2}}, \quad \varepsilon=\frac{1}{r_{d}} \sqrt{\frac{L 11}{C 1}} \\
v_{1}=E x_{1}, \quad i_{11}=E \sqrt{\frac{C_{1}}{L_{11}}} x_{2}, \quad i_{11}=E \sqrt{\frac{C_{1}}{L_{11}}} x_{3}, \\
v_{2}=E x_{4}, \quad i_{21}=E \sqrt{\frac{C_{1}}{L_{11}}} x_{5}, \quad i_{11}=E \sqrt{\frac{C_{1}}{L_{11}}} x_{6},
\end{gathered}
$$

the normalized equations are described as follows.

$$
\left\{\begin{array}{l}
\dot{x}_{1}=\alpha\left(x_{1}+x_{4}\right)-\left(x_{2}+x_{3}\right) \\
\dot{x}_{2}=x_{1} \\
\dot{x}_{3}=\beta_{1}\left(x_{1}-f_{\left(x_{3}\right)}\right) \\
\dot{x}_{4}=\alpha \gamma\left(x_{1}+x_{4}\right)-\gamma\left(x_{5}+x_{6}\right) \\
\dot{x}_{5}=\beta_{2} x_{4} \\
\dot{x}_{6}=\beta_{3}\left(x_{1}-f_{\left(x_{6}\right)}\right)
\end{array}\right.
$$

where

$$
f_{(x)}=\frac{1}{2 \varepsilon}(x+\varepsilon-|x-\varepsilon|)
$$

Here, in order to simplify the analysis, we consider the case that the diode in the circuit is assumed to operate as an ideal limiter [9]. This idealization corresponds to the limit $r_{d} \rightarrow \infty$, i.e. $\varepsilon \rightarrow 0$. When the diode is off, the diode is assumed to operate as open circuit.
When the diode is on, the diode is assumed to operate as $E[V]$ constant voltage source. By using this idealization, circuit dymanics are divided into simplified four piecewise-linear differential equations.

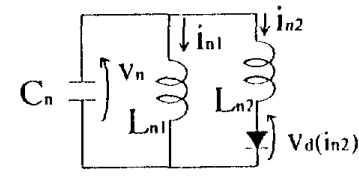

(a)

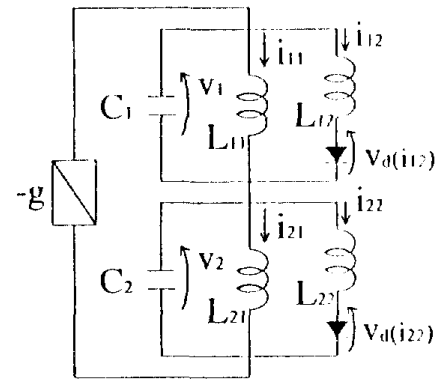

(b)
Fig. 1: Circuit model. (a) Subcircuit. (b) 'Twin chaos generator.

\section{EXPERIMENTAL RESULTS}

\section{Single Subcircuit Case}

We consider single subcircuit case. This circuit is pro posed by lnaba et al [7] and this circuit exhibits a pe riod doubling bifurcation route to chaos. They explained the physical mechanisus of the generation of chaos by changing oscillation frequency [7], [8]. Figure. 2 shows the chaotic attractors and its spectral characteristics obtained by circuit experinents. We observe spectral characteristics in the following conditions.

- FF' effective sample rate : $100.00[\mathrm{kSa} / \mathrm{s}]$

- average : 256

\section{'I'win Chaos}

Next, we consider twin clanos generator. 'This circuit ex hibits chaos on $v_{1}-V_{2}$ plane via torus. Figure. 3 shows the chaotic altractors observed in circuit experinents. In Fig. 3 (1)(a-1), chaotic altractor includes many lower frequency components. However, this chaos includes a few higher frequency components. On the other hand, in Fig. 3 (1)(a-2), chaolic altractor includes many higher frequency components. Figure. 4 shows chaolic attrac tors observed in numerical experiments. We obtained them by using Runge-Kutla method. The numerical re sults match the experimental ones well qualitalively.

Figure. 3 (2) shows spectral characteristics observed in circuit experiments when the circuit exhibits chaos. In ['ig. 3 (2)(a), we can observe chaotic spectrum on lcwer frequency band from $0[\mathrm{~Hz}]$ to about $5[\mathrm{kllz}]$ and periodic one on higher frequency band around 11 [k/lz]. 'This spectrum is similar to one added spectrum in Fig. 2(2)(a) and in F"ig. 2(2)(b). The existence of almost. periodic chaos on higher frequency hand is verified and both of chaos on lower frequency band and ligher frequency band are just about the same. As $\alpha$ increases and 
reaches $\alpha=1.027$, the spectrum become to be chaotic spectrum on the whole (see Fig. $3(2)(b)$ ). However, the spectrum has two crests around lower frequency band and higher frequency band. On higher frequency band, the existence of almost periodic chaos which is not more periodic than chaos in Fig. 3 (1) is verified. Therefore, two chaotic oscillations having quite different frequency components are simultaneously generated in the twin chaos generator.

Moreover, we consider the case the difference of the oscillation frequencies in two subcircuits is small. Circuit experimental results for the case that oscillation frequen-
(1)

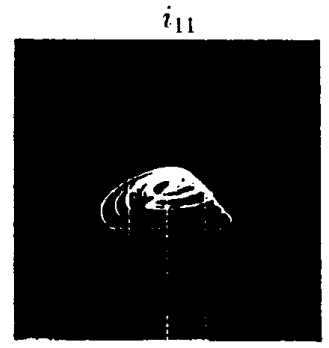

(a)

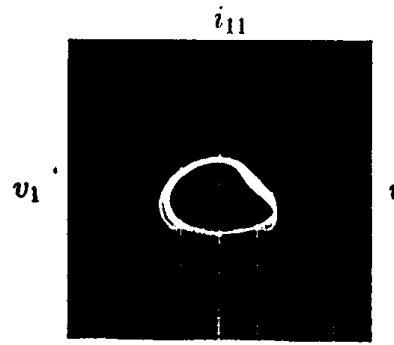

(b)

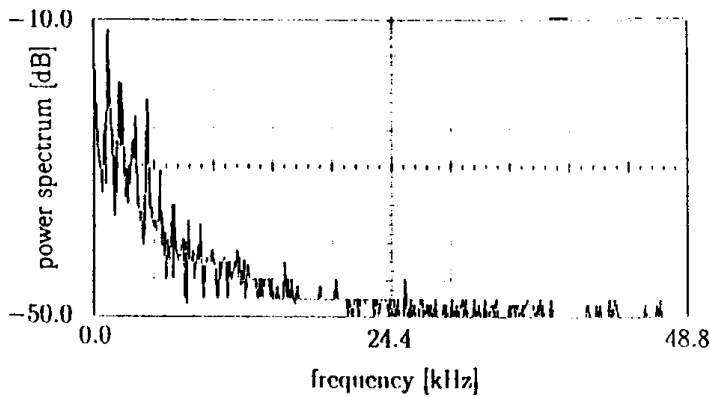

(2)

(a)

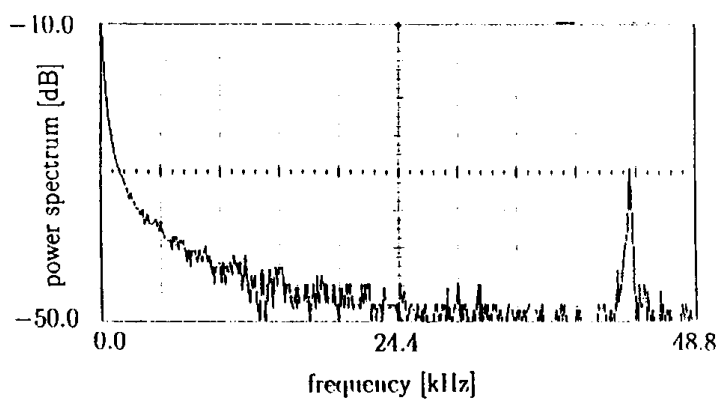

(b)

Fig. 2: Experimental results for single subcircuit case. (1) Chaotic attractor. (2) Spectral characteristics. (a) $\alpha$ $=0.758, \beta_{1}=10.0\left(C_{1}=0.022[\mu \mathrm{F}], L_{11}=500[\mathrm{mH}], L_{12}\right.$ $=50[\mathrm{mH}])$. (b) $\alpha=0.774, \beta_{1}=10.0\left(C_{1}=0.0010[\mu \mathrm{F}]\right.$, $\left.L_{11}=10[\mathrm{mH}], L_{12}=1[\mathrm{mH}]\right)$. cies of two subcircuits are similar are shown in Fig. 5 . By comparison with Fig. 3 (2), each chaotic attractor is affected by higher frequency or lower frequency cornponents. However, spectral characteristics is two crests around lower frequency band and higher frequency band. When two oscillation frequencies are closer, each chaos interferes each other. Finally, twin chaos disappears and chaos which is the same as the case of one subcircuit appears due to mutual leading-in.
(1)

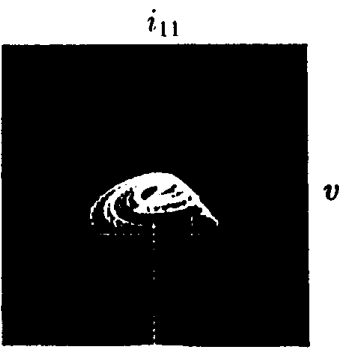

$(a-1)$

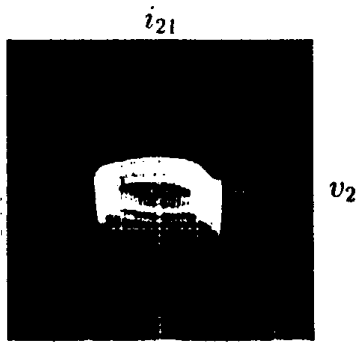

(a-2)

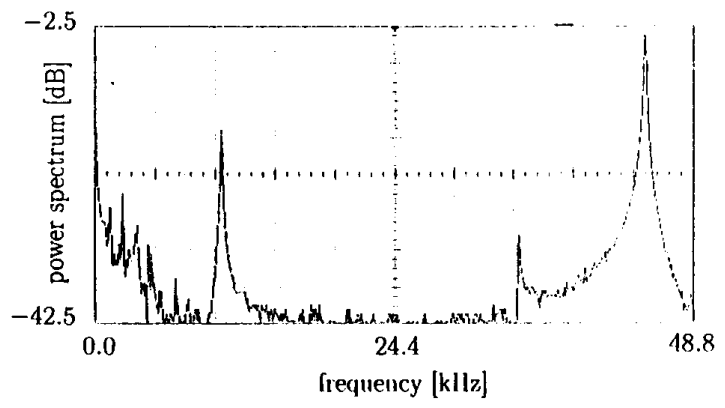

(a)

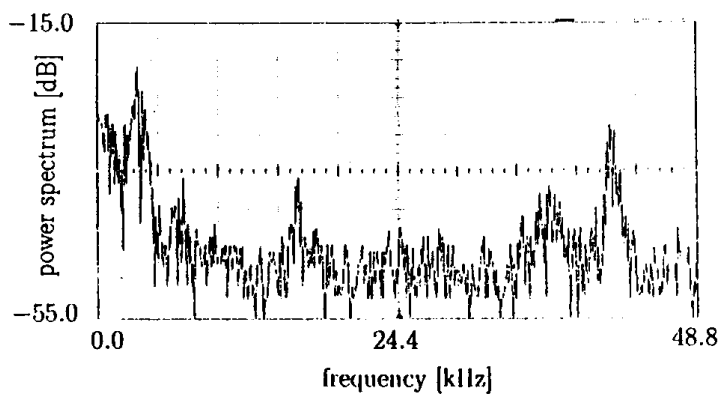

(b)

Fig. 3: Experimental results for twin chaos generator. $\beta_{1}=10.0, \beta_{2}=50.0, \beta_{3}=500.0, \gamma=22.0\left(C_{1}\right.$ $=0.022[\mu \mathrm{F}], L_{11}=500[\mathrm{mH}], L_{12}=50[\mathrm{mH}], C_{2}=$ $\left.0.0010[\mu \mathrm{F}], L_{21}=10[\mathrm{mH}], L_{22}=1[\mathrm{mH}]\right)$ (1) Chaotic attractor. (2) Spectral characteristics. (a-1) Projection onto $v_{1}-i_{11}$. (a-2) Projection onto $v_{2}-i_{21}$. (a) $\alpha=$ 0.745 . (b) $\alpha=1.027$. 

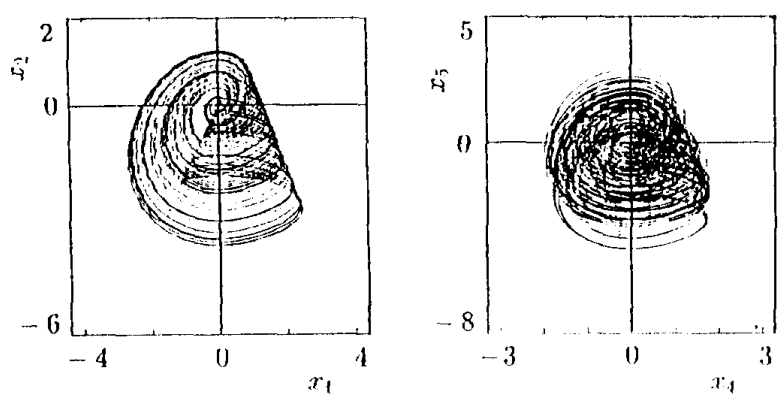

Fig. 4: Numerical results for twin chaos generator. $\alpha=$ $0.591, \beta_{1}=10.0, \beta_{2}=50.0, \beta_{3}=500.0, \gamma=22.0$

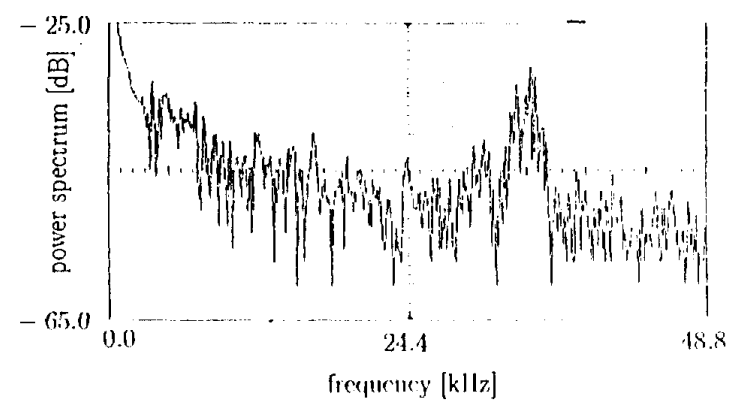

Fig. 5: Spectral characteristics of twin chaos for another parameters. $\alpha=0.701, \beta_{1}=10.0, \beta_{2}=10.0, \beta_{3}=$ $100.0, \gamma=10.0 C_{1}=0.010[\mu \mathrm{F}], L_{11}=100[\mathrm{mH}], L_{12}$ $=10[\mathrm{mH}], C_{2}=0.0010[\mu \mathrm{F}], L_{21}=10[\mathrm{mH}], L_{22}=$ $1[\mathrm{mH}])$

\section{CONCLUSIONS}

In this study, twin chaos generator have been investigated. In the case that oscillation frequencies of two subcircuits are quite different, both subcircuits exhibit asynchronous chaos simultaneously. In circuit experiments we can observe spectral characteristics having two crests on lower frequency band and higher frequency band. We confirm that twin chaos is generated in our circuit. As a results, twin chaos is almost divided into two different kinds of chaos, one includes many lower frequency components and the other one includes many higher frequency components. Moreover as the difference between oscillation frequencies of two subcircuits decreases, two different kinds of chaos interfere mutually. Finally, twin chaos disappears and chaos which is the same as the case of one subcircuit appears due to mutually leadingin. In order to obtain stable asynchronous $N$ chaos having different oscillation frequencies simultaneously, we must choose quite different oscillation frequencies of $N$ subcircuits. Simultaneous asynchronous $N$ chaos having different oscillation frequencies are generated stably in our system if it is possible that the interference is almost ignored. Therefore, We believe that chaos generated in our system is almost divided into $N$ kinds of chaos and this results would give the effective sugges tions to make clear the nonlinear phenomena in higher dimensional systems coupled same chaotic circuits.

\section{REFERENCES}

[1] B. van der Pol, "An oscillation hysteresis in a triode generator with 2-degrees of freedom," Phil. Mag., Vol. 43, pp. 700-719, Apr. 1922.

[2] B. van der Pol, "The nonlinear theory of electric oscillations," Proc. IRE., Vol. 22, pp. 1051-1086, Sept. 1934.

[3] J.S. Schaffner, "Simultaneous oscillations in oscillators," IRE Trans. Circuit Theory., Vol. CT-1, pp. 28, June. 1954.

[1] M. I. Disman and W. A. Edson, "Simultaneous asynchronous oscillations in class-C oscillators," Proc. IRE., Vol.46, pp. 895-903, May. 1958.

[5] 'T. Saito, T. Takagi and K. Mano, "Asynchronous Multimode Oscillations in Negative Conductance Oscillator with Nonlinear Resonators," Trans. IECE., Vol. 57, A No. 7, pp. 511-518, July. 1974. (in Japanese).

[6] T. Matsumoto, L. O. Chua and M. Komuro, "Ihe Double Scroll," IEEE Trans. Circuit Syst., CAS-32, No. 8, pp. 797-818, Aug. 1985.

[7] N. Inaba and S. Mori, "Chaotic Phenomena in a Circuit with a Diode due to the Change of the Oscillation Frequency," Trans. IEICE, Vol. E7I, No. 9, pp. 842-849, Sep. 1988.

[8] N. Jnaba and T. Saito, "On Physical Mechanisms of Chaos in a Third Order Autonomous Circuit Family with a Diode," Trans. IElCE, Vol. J74-A, No. 12, pp. 1766-1773, Dec. 1991. (in Japanese).

[9] N. Inaba, 'I'. Saito and S. Mori, "Chaotic Plienomena in a Circuit with a Negative Resistance and an Ideal Switch of Diodes," Trans. IEICE., Vol. E70, No. 8, pp. 744-754, Aug. 1987.

[10] T. Matsumoto, L. O. Chua and K. Kobayashi, "Hyperchaos: Laboratory Experiment and Numerical Confirmation," IELE Trans. Circuit Syst, CAS-33, No. 11, pp. 1143-1147, Nov. 1986.

[11] T'. Saito, "An Approach Toward Higher Dimensional Hysterisis Chaos Generators," IEEE Trans. Circuit Syst., CAS-37, No. 3, pp. 399-409, March. 1990. 